ISSN: $1130-3743$

\title{
LA ANTROPOLOGÍA DE LA EDUCACIÓN COMO DISCIPLINA: PROYECTO DE DISEÑO
}

\section{EDUCATIONAL ANTHROPOLOGY AS DISCIPLINE: AN PROJECT OF DESIGN}

HENRI BOUCHÉ PERIS, PACIANO FERMOSO ESTÉBANEZ, JORGE LARROSA BONDÍA, DAVID SACRISTÁN GÓMEZ (+)

Universidad Nacional de Educación a Distancia, Departamento de Teoría e Historia de la Educación y Pedagogía Social. Facultad de Educación. Senda del Rey, s/n., 28040 Madrid; Universitat Autónoma de Barcelona. Departament de Pedagogía Sistemática i Social. Edifici B, 08193 Balleterra, Barcelona; Universitat de Barcelona. Departament de Teoría i Historia de la Educación. Baldiri Reixac, s/n 08208 Barcelona.

RESUMEN

Los fundamentos antropológicos de la educación han sido objeto de reflexión y estudio desde Sócrates hasta nuestros días. Pero su sistematización como un elemento del currículum de Ciencias de la Educación no tiene una historia tan larga. Gran parte de su desarrollo se produjo en la segunda mitad del siglo XX gracias al trabajo tanto de la comunidad filosófica como de la comunidad científica. En particular, ha sido en Alemania y en los Estados Unidos de América donde se ha configurado el campo, aunque desde diferentes perspectivas y utilizando diversas terminologías.

Sin embargo todavía persiste una doble aproximación, filosófica por un lado y científica por otro, ambas reflejadas en la materia oficialmente promovida por el MEC para su impartición como materia troncal en los nuevos títulos ofrecidos en el nivel universitario.

Un estudio empírico nos mostrará cómo las distintas Universidades españolas están configurando sus programas. Por otra parte, y a partir de aspectos relevantes de la Historia, la Epistemología, la Administración Educativa, y también de sus propias experiencias universitarias, los autores someterán a la consideración de la comunidad universitaria una propuesta de Diseño de Programa que no será, en absoluto, ni la primera ni la mejor. 
SUMMARY

The anthropological roots of education have been the subject of reflection and investigation since the time of Socrates up to the present day. But its systemisation as an element of the curriculum of Education Sciences does not boast such a long history. Indeed most of its development ocurred in the latter half of the 20th century, thanks just as much to the work of the philosophical community as the scientific. Germany and the United States of America in particular have led the field in related studies, although from different perspectives and using different terminology.

Nowadays, there is still a double aspect to the approach to this subject: philosophic on the one hand scientific on the other, both of which are reflected in the subject matter officially promoted by the MEC for use in foundation (spanish; "troncal") courses in the new degrees offered at university level.

An empirical study will show us how spanish Universities are settting up their own programmes. Based on relevant aspects of History, Epistemology, Educational Administration and the Universities own experiences, the authors will undertake the submission of a Programme Design proposal, which will not, by any means, be the first of its kind, not the most perfect.

El estudio que ofrecemos al lector es la Ponencia, revisada para ser publicada en esta Revista interuniversitaria, que los autores presentamos en el "XIII Seminario Interuniversitario de Teoría de la Educación" (Tarragona: diciembre 1994). No pretendimos otra cosa que elaborar un "Proyecto", tal vez, porque en el fondo no se pueda, ni se deba hacer más. Pero un "Proyecto", por más que haya de ser revisable, por su misma naturaleza, supone, sin embargo, una toma de postura.

Todo Proyecto, éste también, es un documento de trabajo, en el que previamente se han ordenado, estudiado y elaborado los datos y los supuestos básicos de una determinada cuestión, a fin de ofrecer, como síntesis, una propuesta concreta, abierta y sugerente, que pueda ser nuevamente estudiada.

El presente es uno entre los múltiples posibles Proyectos/Diseños de la asignatura "Antropología de la Educación", troncal de cuatro créditos, en el nuevo Plan de Estudios de Pedagogía, en las Universidades españolas.

Los autores no han tenido otra pretensión que aportar sugerencias, con la intención de evitar excesiva dispersión en el tratamiento de una disciplina académica, que comienza. En ningún momento han pretendido suplantar la acción de los Departamentos, ni de las Facultades, ni de las Universidades, ni la libertad de cátedra, privilegio y derecho inherente a la docencia universitaria, sino responder al encargo que nuestros colegas, en este contexto tolerante, nos hicieron.

La estructura de la Ponencia abarca tres apartados: justificación epistemológica, consideración empírica y propuesta de programa. En el primero de ellos, el más discutible, se fundamenta su estatuto epistemológico; en el segundo, recogemos los datos empíricos descriptivos y comparados entre los programas que, de hecho, algunos compañeros han confeccionado y los descriptores principales de 
HENRI BOUCHÉ PERIS, PACIANO FERMOSO ESTÉBANEZ, JORDI LARROSA BONDÍA, DAVID SACRISTÁN GÓMEZ (+)

algunas obras, clásicas ya, en esta materia, publicadas más allá de nuestras fronteras; finalmente, en el tercero, hacemos una propuesta concreta, con los núcleos temáticos fundamentales que podrían estudiarse en la "Antropología de la Educación" ateniéndonos a tres fuentes: el estatuto espistemológico, la descripción empírica y las materias sugeridas por el propio MEC, al aprobar las troncales de la nueva Licenciatura en Pedagogía, y los contenidos de los programas recibidos.

\section{Estatuto EpISTEMológico de la ANTROpología de la Educación}

\section{1) Antecedentes de la Antropología de la Educación}

Los antecedentes han de buscarse en las relaciones de la educación y de la Pedagogía con la Antropología. Es evidente, en efecto, que tanto la acción educativa practicada desde los griegos, como el quehacer pedagógico presente, fundamentado en bases científicas, han supuesto una determinada concepción antropológica, más o menos explícita. Esta tesis tiene validez, sobre todo, en las investigaciones más recientes acerca de las mutuas relaciones entre la Pedagogía y la Antropología. Es un hecho comprobable en la Filosofía de la Educación e incluso en la Pedagogía General, en la que sus supuestos son filosóficos también, como secuela de la postura herbartiana, que radicaba el saber científico sobre educación en la Etica y en la Psicología ("racional", es decir, filosófica, en aquellas calendas). Más palpable es aún esta radicación en el caso de la Filosofía de la Educación, que ha sido para algunos "Fundamentos filosóficos de la educación", "Sistemas educativos" o "Filosofía para educadores".

Entre las disciplinas pedagógicas, que han contemplado la educación desde perspectivas antropológicas, cabe destacar tres, por su tradición y difusión: la "Antropología Pedagógica", la "Pedagogía Antropológica" y la "antropología social o cultural de la Educación". Las precisiones epistemológicas se han fijado en la sustantividad o adjetividad de las dos primeras.

$1^{\text {a) }}$ La Antropología Pedagógica. En Alemania ha sido la perspectiva más habitual, defendida por prestigiosos pedagogos y filósofos, que la han considerado "una disciplina pedagógica, científicamente fundamentada", "síntesis de relevantes conocimientos educativos, aportados por la Psicología, la Sociología, el Psicoanálisis, la Ciencia de la conducta, la Antropología cultural y otras ciencias humanas" (Zdarzil: 1975, 776). Uno de esos prestigiosos científicos es H. Roth, que la califica de "ciencia integradora de las cuestiones pedagógicas" (citado por Zdarzil: 1975, 776). Hasta es tenida por "caduca e inútil la reivindicación de su carácter autónomo entre las disciplinas científicas de la educación". H. Zdarzil, posiblemente uno de los pedagogos con más clarividencia epistemológica, la considera una "ciencia, filosófica y empírica a la vez" (Zdarzil: 1975, 783).

Esta primera perspectiva sostiene que la "Antropología Pedagógica" es inseparable de la Antropología General; y que se trata de una disciplina antropológica, no pedagógica, o sea, que no es un saber normativo. 
HENRI BOUCHÉ PERIS, PACIANO FERMOSO ESTÉBANEZ, JORDI LARROSA BONDÍA, DAVID SACRISTÁN GÓMEZ (+) LA ANTROPOLOGÍA DE LA EDUCACIÓN COMO DISCIPLINA: PROYECTO DE DISEÑO

$\left.2^{a}\right)$ Pedagogía Antropológica. En contraposición a la perspectiva anterior, ésta es un saber normativo, un saber pedagógico. Su finalidad es llegar a descubrir características humanas a través del fenómeno educativo.

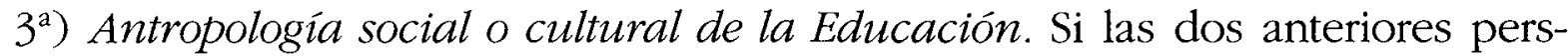
pectivas son básicamente germanas, ésta es anglosajona; es la postura más comúnmente admitida en Estados Unidos y en el Reino Unido, donde consideraciones filosóficas aparte, ha predominado la perspectiva culturalista (Fermoso: 1994, 78-81).

\section{2) La Especifidad de la Antropología de la Educación}

Implícita en las tres perspectivas mencionadas descubrimos otra forma de resolver el problema: nos preguntamos si la Antropología de la Educación es una disciplina filosófica o científica; si existen una o varias modalidades.

Si hubiéramos de localizar geográficamente las respuestas y propuestas a este problema, diremos que la identificación con una disciplina filosófica se ha defendido en Alemania, principalmente por fenomenólogos; la identificación con una disciplina científica ha prevalecido preferentemente en EE.UU. y el Reino Unido, aunque también ha habido cultivadores de esta postura en Alemania, donde ha sobresalido H. Roth (Roth: 1966). Y finalmente la postura integradora es la ya indicada de Zdarzil, con quien comparten su opinión algunos pedagogos castellanoparlantes, cuyos nombres no pueden omitirse: Nassif, Mantovani y Bertrán Quera.

Aunque en los descriptores y las materias que el MEC menciona en el Decreto sobre la nueva titulación de Pedagogía, hay módulos filosóficos y de Antropología cultural, no puede deducirse de aquí que se posicione epistemológicamente a favor de la perspectiva integradora, sino que se ha adoptado una solución "salomónica" y complaciente con los defensores de la perspectiva filosófica y la científica.

Podemos hacernos éstos y parecidos interrogantes; ¿Qué supone la Antropología de la Educación? ¿Qué añade o en qué supera la Antropología de la Educación a lo aportado por la Antropología general filosófica y por las Antropologías científicas? ¿Es realmente posible una integración de las distintas Antropologías, desde la formalidad educativa? Y de ser ésta factible, ¿por qué modalidad inclinarnos?

Se ha intentado ya, aunque sin fortuna, fusionar los conocimientos filosóficos y científicos sobre el hombre, porque parecen irreconciliables los estudios científicos y los filosóficos; unos abominan de los otros, herencia del positivismo y del "complejo" generalizado entre los filósofos, y sobre los que se piensa han prestado escasos servicios a nuestro mundo utilitario y eficaz. Una de las posibles causas ha sido la multiplicidad de ciencias humanas, que permiten repetir la postura escéptica y desilusionada de Max Scheler:

"Nunca el conocimiento del hombre ha sido tan problemático como en nuestros días y, por otro lado, nunca se ha mostrado tan urgente. Es necesario establecer un amplio diálogo interdisciplinar entre teoría y científicos positivos buscando 
la clarificación de lo humano. La clarificación de un auténtico saber comprensivo del hombre no puede ser tarea de una actividad aislada" (Scheler, citado en Escamez: 1981, 8).

No sería aceptable, epistemológicamente hablando, concebirla como un saber interdisciplinar, con niveles cognitivos distintos: filosófico y científico. La interdisciplinariedad no permite franquear los límites entre niveles diferentes. Puede recurrirse a la interdisciplinariedad, en el ámbito filosófico, por ejemplo, configurando una imagen del hombre ética, psicológica y ontológica; y es permisible también entre la Biología, la Sociología, la Etnografía, etc. No sería desacertado, sin duda, que el educador conciba integralmente al hombre, pero consciente de que no pueden fusionarse el conocimiento empírico y el filosófico; sí coordinarse y completarse.

Los antropólogos de la educación se mueven dentro de las concepciones y del espacio científico sobre la cultura; la Antropología de la Educación es una subdisciplina de la Antropología de la Cultura, que entiende sobre su adquisición y transmisión, tal y como han puesto de manifiesto los antrólogos norteamericanos, desde 1954, y como hemos historiado recientemente (Fermoso: 1994, 79; GarcíaCastaño y Pulido: 1994). La metodología es etnográfica y se ha aplicado en investigaciones microsociológicas, en especial en el estudio de las interrelaciones escolares, expectativas profesor/alumnos, alumnos/profesor, etc.

Por paridad, la Antropología de la Educación, desde la perspectiva filosófica, es una subdisciplina de la Filosofía de la Cultura, con solera y raíces profundas en la filosofía alemana neokantiana y otras.

Pero resulta incuestionable que no existe aún una concepción antropológica -ni filosófica, ni empírica- capaz de satisfacer y de convencer suficientemente a la comunidad científica, por lo que el diseño/proyecto de la Antropología de la Educación tampoco podrá ir más allá, por el momento, del reconocimiento de una pluralidad de modelos antropológicos y de manifestaciones culturales igualmente distintas. Desde cada una de ellas se ofrece una base, igualmente diferente, del quehacer educativo, sometido a normatividad y al "ser ideal" del hombre. Filosófica o científicamente ha de construirse un concepto del hombre, al cual se ajuste la praxis. Nada puede hacerse, en Pedagogía, sin la Antropología; educación y Antropología ha caminado paralelamente, en mutuo diálogo, porque como afirmó Kant, "el hombre sólo llega a ser hombre a través de la educación" (Kant: 1991, 8).

La doble vía y la pluralidad en cada una de ellas no deben conducir a un fácil relativismo antropológico; antes al contrario, las plurales concepciones sobre el hombre habría que entenderlas como una normal secuencia de los distintos momentos del desarrollo de la investigación, compleja y polifacética, de modo que quien se dedique a estos menesteres ha de distinguirse por una especial dedicación intelectual, por una contrastada apertura mental y por una alta dosis de perseverancia en su trabajo. Sólo así podrán evaluarse las distintas hipótesis explicativas de lo humano, al no conocerse aún su alcance. Es un hecho que los trabajos de Antropología de la Educación corren paralelos a las clásicas grandes concepciones sobre el hombre: la del homo sapiens, repetida en el mundo occidental y cristiano; la del bomo faber, imagen naturalista-biologista; la del bomo laborans, 
preferida por el marxismo. Y los más recientes, tienen en cuenta las modalidades postreras de la Antropología, como hace, por ejemplo, Hamann en su obra, vertida poco ha al castellano (Hamann: 1992), en la que recoge las propuestas antropológicas de Max Scheler, N. Hartmann, A. Gehlen, A. Portmann, el Conductismo, la Cibernética, el Psicoanálisis, la Psicología Humanista, la Logoterapia de V. Frankl, etc.

El problema no es nuevo. Ya Max Scheler se había hecho un planteamiento casi idéntico, en las dos obras (El puesto del hombre en el cosmos; La idea del bombre y la bistoria) en las que recogió el material que iba a ser el esbozo de un gran proyecto antropológico, truncado por su muerte. En ellas comenta Scheler que si se prescinde de las antropologías prefilosóficas y prelógicas, las concepciones más influyentes en occidente han sido las clásicas ya mencionadas, más la del superhombre de Nietzsche. En El puesto del hombre en el cosmos, con manifiesta intención integradora, advierte que se propone "un ensayo de antropología filosófica de más amplia base... en la que quisiera dilucidar algunos puntos concernientes a la esencia del hombre en relación con el animal y con la planta, y al singular puesto metafísico del hombre... pues la misión de la Antropología filosófica es mostrar exactamente cómo la estructura fundamental del ser humano, entendida en la forma en que la hemos descrito en las consideraciones anteriores, explica todos los monopolios, todas las funciones y obras específicas del hombre "el lenguaje, la conciencia moral... y la sociabilidad" (Scheler: 1971).

En todo caso, para nuestro cometido, lo realmente importante son las coincidencias y los acuerdos, que permiten establecer un núcleo de problemas comunes, que orienten al estudioso de la Antropología de la Educación; es decir, interesa destacar las caracterizaciones antropológicas - filosóficas y empíricasaceptadas en nuestros días, con cierto acuerdo mayoritario. Pensamos, a pesar de todas las dificultades y discrepancias, que es más práctica una actitud integradora, que la adhesión unilateral a una determinada concepción antropológica.

\section{3) La Antropología de la Educación como Antropología en los contextos pedagógicos}

Somos conscientes de que la Antropología de la Educación puede ser entendida en el marco de contextos antropológicos o en el de contextos pedagógicos; estos últimos son, por lógica, los que nos interesan. Pero aún en estos contextos distinguimos: la Antropología de la Educación como conjunto de teorias antropológicas de la educación: y la Antropología de la Educación como conjunto de tópicos antropológicos, que tienen algún interés para la Pedagogía.

3.1.-La Antropología de la Educación como conjunto de teorias antropológicas de la educación. La Antropología de la Educación, en este primer sentido, se distingue de otras especialidades antropológicas por el ámbito y la realidad a la que se aplica; y de otras teorías de la educación, porque está construida con herramientas de carácter antropológico. En una palabra, la Antropología de la Educación es, en cuanto teoría, una de las muchas concebidas sobre el proceso educa- 
tivo; sin embargo, las fronteras entre ella y otras antropologías especiales, y entre ella y otras concepciones pedagógicas no son precisas.

En la Antropología de la Educación, como teoría educativa, distinguimos un doble aspecto: $1^{\circ}$ ) Exégesis de textos antropológicos sobre educación, interpretados a la luz de supuestos generales en toda antropología, a fin de delimitar el proceso educativo y de apreciar las distintas escuelas que constituyen el panorama antropológico contemporáneo. Esta modalidad es una operación deductiva, porque se construye una teoría de la educación, derivándola de la Antropología general. $\left.2^{\circ}\right)$ Explicación de los supuestos antropológicos, implícitos en las propuestas educativas, o sea, desde las pedagogías carentes de considerandos antropológicos expresos. Esta modalidad o aspecto de la Antropología de la Educación tiene carácter operativo y permite someter a examen todas las concepciones pedagógicas.

Lo específico de la Antropología de la Educación, como teoría educativa es la tematización de las realidades y de la praxis pedagógicas.

3.2.-La Antropología como conjunto de tópicos antropológicos de interés pedagógico. Es ésta una concepción instrumental, contrapuesta a la teórica tematizadora; se trata de investigar las relaciones particulares entre Antropología y Pedagogía en contextos particulares. Supone una concepción pragmática de la investigación pedagógica, con énfasis especial en el proceso productivo del conocimiento y como una práctica social propositiva y más o menos organizada de transformación cognitiva. El conocimiento, en este caso, es entendido holísticamente, estrategia común a la epistemología postpopperiana; por ejemplo, el concepto de "paradigma" en Kuhn, o el de "poblaciones conceptuales" en Toulmin, o el de "red cognitiva" en Hesse, o el de "complejo de conocimientos, problemas e instrumentos" en Radnitzky. La investigación pedagógica, así entendida, produce y transforma un conjunto heterogéneo de supuestos ontológicos y epistemológicos, problemas y estrategias herurísticas, teorías, metodologías de trabajo, modelos y analogías, etc.

Esta concepción pragmática de la Antropología de la Educación considera a estos componentes intelectuales como herramientas usadas en determinados contextos para hacer determinadas cosas; es decir, los componentes intelectuales se usan en un contexto empírico y social (Radnisky: 1974). En esta forma de entender la Antropología de la Educación interesan la utilización y circulación de las ideas, o su equivalente, cómo, para qué, y qué se hace con ellas; forma parte del "mercado de recursos intelectuales", que presta una ayuda y auxilio, para decir "algo a alguien" (Rorty: 1983), que recurre a ella para la realización de actividades específicas y selecciona los contenidos antropológicos considerados útiles dentro de un contexto concreto.

\section{4) La Antropología de la Educación, como asignatura/materia y comunidad científica de especialistas}

En el mundo académico, una disciplina puede entenderse de tres modos: $1^{\circ}$ ) Como asignatura, es decir, como cuerpo de conocimientos organizado desde la 
perspectiva de la adquisición y de la transmisión; $2^{\circ}$ ) Como materia o contenido intelectual elaborado con criterios teóricos; y $3^{\circ}$ ) Como comunidad de especialistas o grupo de personas institucionalizado, cuyo contenido es la producción, uso y transmisión de conocimientos. Estos tres modos de entender una disciplina manifiestan su dimensión social, su dimensión pedagógica y su dimensión intelectual.

La Antropología de la Educación ha comenzado a configurarse como una asignatura troncal en los Planes de estudios pedagógicos; por lo tanto, interesa su adquisición y su transmisión, en un contexto pedagógico. Toda asignatura requiere, como soporte intelectual, un corpus heterogéneo de textos y autores, un conjunto delimitado de conceptos, teorías, métodos, temas y problemas. Y, por último, requiere también el soporte social de un colectivo profesional, universitario en nuestro caso, con determinadas funciones, que necesita un reconocimiento.

En cuanto asignatura, tiene que configurar cierto espacio en los planes de estudio pedagógicos y tiene que fijar objetivos encaminados a la formación de educadores y pedagogos. En cuanto materia, ha de asumir cierta responsabilidad en la construcción, administración, uso y ampliación de su propio contenido intelectual. Y en cuanto comunidad científica profesional en contextos pedagógicos, ha de conquistar un lugar en los canales de circulación del discurso pedagógico (revistas, bibliografías, foros formales e informales de comunicación) y ha de desempeñar un papel en la construcción del conocimiento pedagógico.

Como asignatura, la Antropología de la Educación ha de delimitar los objetivos, ha de elaborar un programa con una selección y secuenciación en los contenidos, y ha de sugerir los procedimientos de la enseñanza y del aprendizaje. El programa, por ejemplo, ha de adaptarse a la duración del curso académico y a los créditos asignados, lo que exige seleccionar los temas, textos y autores, a la vez que se justifica dicha selección con una mezcla de criterios, que evidencian el significado de la asignatura en el Plan de estudios, la formación previa, los intereses y preocupaciones de los alumnos. En cuanto asignatura exige conocimientos de Antropología general y las exigencias del contexto formativo universitario, en el que se inscribe.

Como materia, la Antropología de la Educación ha de abarcar todo lo que sea significativo, de modo que, a partir de él, puedan generarse distintas propuestas curriculares concretas, según los cursos y los diversos niveles de especialización, teniendo presentes sus relaciones con otras Antropologías especiales y con otras materias pedagógicas.

Como comunidad científica de profesionales, la Antropología de la Educación, en contextos pedagógicos, ha de explicitar las funciones de quienes integran el colectivo. Los tres modos de entenderla exigen algunas operaciones previas de cierta complejidad: qué es la Antropología general; su estructuración interna; la problemática de la educación como objeto de trabajo; el carácter interdisciplinar de la Pedagogía; y cómo se entiende su enseñanza.

Las ideologías de las profesiones intelectuales o "ideologías disciplinarias" han sido investigadas por la epistemología postkhuniana. Hay quien las entiende como un conjunto explícito de afirmaciones sobre las características genera- 
les y sus objetivos, requisito previo para la autoidentificación de los miembros de una comunidad científica (Hagtroms: 1965). A la luz de las exigencias propias de las ideologías generadas por toda profesión, la Antropología es, entre otras cosas, una institución, tal y como han probado serias investigaciones (Bourdieu, 1985; Foucault: 1973; Kuhn: 1971). Esto significa que la Antropología de la Educación es una actividad colectiva de grupos de índole social homogénea, sometida a reglas explícitas y rigurosas y cuyo producto - sistemas, conceptos, teorías, etc.- es compartido y administrado por los componentes de un colectivo.

La Antropología de la Educación, en consecuencia, tiene dependencia de los grupos sociales que la trabajan, la administran y la transmiten; es difícil separar su componente intelectual, de su componente institucional, y la dinámica sociológica, de la dinámica intelectual, tal y como ha probado la investigación epistemológica con sensibilidad sociológica (Knorr-Cetina y Mulkay: 1983). La Antropología de la Educación ha de buscar un "espacio propio" en el mundo intelectual, ha de especificar su identidad y diversidad y ha de hacerse respetar en los ambientes universitarios. En pocas palabras, ha de legitimarse institucionalmente, mediante la demostración práctica de que quienes la cultivan comparten ciertos rasgos intelectuales y están sometidos a ciertas reglas de trabajo. Sólo así podremos hablar de la Antropología de la Educación como de una especialidad y de una actividad organizada y disciplinada.

La ubicación institucional de la Antropología de la Educación guarda correspondencia con dos tipos de comunidad científica. El primer tipo es una comunidad relativamente cerrada, homogénea y cohesionada, porque sus estudiosos e investigadores leen a otros antropólogos, se plantean problemas con tradición cultural y se dirigen básicamente, en sus escritos y comunicaciones, a otros especialistas de su mismo ámbito. El contexto social del conocimiento da gran importancia a la audiencia y/o lectores especializados (Witley: 1984).

El segundo tipo está formado por antropólogos de la educación, que trabajan en contextos más plurales y heterogéneos o en comunidades científicas no estrictamente antropológicas, en las que se modifican el contexto social, las formas solidarias, la identidad intelectual y profesional, el marco axiológico, las creencias compartidas y los intereses. La Antropología de la Educación, así concebida, deja de ser una comunidad científica cerrada y se hace más permeable a las condiciones socioculturales de esos contextos. La consagración a la Antropología de la Educación, en este segundo tipo, no es total, sus investigaciones y lecturas no son exclusivamente antropológicas, se plantea problemas extraños a esta ciencia y se dirige a audiencias más heterogéneas.

En cualquier caso, no interesa analizar sólo qué es la Antropología de la Educación, sino cómo se instala y se desarrolla en diferentes contextos intelectuales y sociales. La configuración de esos contextos determina el contenido intelectual compartido y el tipo de problemas con los que trabaja y las reglas de creación, evaluación y uso del conocimiento producido. 


\section{5) La Comunicabilidad Académica de la Antropología de la Educación}

En la filosofía presocrática y socrática se discutió si los saberes podían ser comunicados. Los innatismos platónico, agustiniano, etc. se han opuesto a la comunicabilidad del saber, mientras que los empirismos, el inglés, por ejemplo, le han sido favorables, porque el aprendizaje lo justifica. El tema reverdeció en el Análisis Lógico, pues se ha dicho que las estructuras verbales la posibilitan. Edmundo Husserl dedicó sustanciosas páginas a rebatir al sofista Gorgias, defensor de la incomunicabilidad del conocimiento (Ayer: 1962; Husserl: 1942).

La comunicabilidad de los saberes se traduce hoy en términos de enseñanza/aprendizaje. Aplicada a nuestra asignatura/materia, equivale a hablar de si y cómo debe plantearse su docencia. Es una consecuencia lógica del estatuto epistemológico de la Antropología de la Educación, aunque, en puridad cognitiva, se trate del resultado didáctico del problema epistemológico. Partimos de la necesidad de los dos polos en todo acto didáctico: profesor/alumno. Prescindimos de la cuestión previa, relativa a la formación inicial y permanente del profesorado de Antropología de la Educación, que ha de ser, por un tiempo, autodidáctica, posible si sus conocimientos pedagógicos y antropológicos son suficientes para establecer la interdisciplinariedad y aplicación.

Importa clarificar el papel que esta asignatura tiene en la formación inicial de los educadores y pedagogos, que no especialistas, docentes o profesionales, al modo norteamericano (Fermoso: 1994). Esta disciplina ha de capacitar a educadores y pedagogos par entender, more antbropologico, las cuestiones educativas, con capacidad reflexiva y crítica.

Veamos, en primer lugar, qué significa aprender Antropología de la Educación. En principio, se adquiere un "cuerpo de conocimientos" y de "habilidades técnicas"; es decir, se aprende una tradición institucionalizada, cuyos contenidos son determinados textos, autores, temas, conceptos y teorías, que existen en los contextos pedagógicos.

La posesión, la administración, la ampliación y el uso de ese "depósito" sería el principal trabajo de los antropólogos de la educación, junto con su reproducción crítica. La transmisión de ese cuerpo de conocimientos sería la principal tarea de los profesores de Antropología de la Educación. Desde ese punto de vista, la enseñanza de la Antropología de la Educación consistirá básicamente en la capacidad del profesorado para presentar ese depósito como algo a ser conocido y reconocido en los contextos pedagógicos, y para asistir a los alumnos en las lecturas, la asimilación y la crítica del "corpus" que constituye ese depósito. Un buen profesor de Antropología de la Educación es aquél que la conoce bien y sabe comunicarla; o sea, ha de ser un experto.

Desde el punto de vista del alumno, la Antropología de la Educación es algo que hay que llegar a saber; básicamente es el conjunto de textos y autores, que, por tradición, son obligada lectura para quien se propone saber Antropología de la Educación; se trata, en realidad, de acumular, de haber leído y conocido más, con tal de que se haya asimilado y verificado un aprendizaje significativo. Dicha acumulación de conocimientos es controlable, pues afecta a la extensión y a la asi- 
HENRI BOUCHÉ PERIS, PACIANO FERMOSO ESTÉBANEZ, JORDI LARROSA BONDÍA, DAVID SACRISTÁN GÓMEZ ( + )

milación, es decir, a una habilidad para ordenar, clasificar y relacionar contenidos antropológicos, implícitos en el proceso pedagógico.

Bien diferente es la adquisición de cierta competencia en una actividad profesional, que no es una acumulación de textos y autores, ni es fácilmente controlable, aunque podamos distinguir cualitativamente una mayor o menor habilidad.

Pensar antropológicamente es la capacidad de desarrollar crítica y reflexivamente una posición antropológica sobre cuestiones educativas, aunque, en un principio, esté llena de dudas y lagunas. Esta capacidad implica una determinada manera de percibir dichas cuestiones y una determinada manera de argumentar en torno a ellas.

¿Qué entendemos por una "posición antropológica"? Distinguimos dos significados: $1^{\circ}$ ) Una posición antropológica entendida como una "posición particular" o doctrina, de más o menos alcance, sobre cualquier tópico antropológico educativo específico; y $2^{\circ}$ ) Una posición personal, que lleva consigo presuposiciones paradigmáticas muy generales, que pueden estar implícitas en una variedad de creencias sobre tópicos antropológicos educativos particulares y en una gran variedad de conductas pedagógicas. El segundo significado está menos explícitamente formulado y será más incierto, porque este tipo de posición se "predica" más de los individuos que de los argumentos por ellos defendidos.

La diferencia entre estos dos significados de la "posición antropológica" consiste en su grado de generalidad y en su grado de explicitación. Cuando se explicita y se argumenta respecto a un ámbito restringido, la posición personal se convierte en una posición particular.

Somos de la opinión que aprender a pensar antropológicamente, tal como puede hacerse en un contexto académico, tiene que ver, sobre todo, con adquirir posiciones antropológicas particulares; aunque otra cosa bien distinta es formar posiciones antropológicas personales. El contexto académico se caracteriza por exigencias de racionalidad y de argumentación, posibilitadas por las "herramientas" teóricas específicas; este contexto obliga a moverse en un nivel de posiciones más explícitas y mejor argumentadas, al dominar el contenido intelectual de la materia. El contexto académico exige que cada participante tenga su propia Antropología de la Educación y que trabaje técnica y conceptualmente con mayor precisión, aunque no se puedan eliminar totalmente las posiciones personales de los participantes.

Una posición antropológica, en cualquiera de los dos sentidos atribuidos, no sólo tiene que ver con aspectos lógicos, pues intervienen también las actitudes básicas de las personas. Implica, por tanto, un irreductible componente decisional. Si se tratara únicamente de aspectos lógicos, se podría tener sólo una posición para cada tópico particular y todas las demás posiciones posibles deberían ser ilógicas, pseudoposiciones o posiciones absurdas, de obligada corrección. La lógica afecta a la coherencia interna de una posición y al modo de argumentarla, pero no será jamás un tribunal de apelación respecto a la posición antropológica misma.

Esto no significa, sin embargo, que desarrollar o adoptar una posición antropológica sea algo independiente de la argumentación; ni que se adquiera por una 
elección, hecha informándose de las distintas posiciones posibles y escogiendo la que a uno más le gusta, como en una suerte de supermercado de la ideas. La posición antropológica no es consecuencia de la argumentación, sino que es efecto de la producción, del contraste y del aprendizaje de argumentos favorables y desfavorables.

En este sentido, una posición antropológica se revela a uno mismo y se constituye solamente si se argumenta en ella, porque no hay otra vía para desarrollarla, que expresarnos en el mundo y reconocernos en y a través de esa expresión. Puede lograrse también mediante el reconocimiento de los otros en un aprendizaje mediado por la intersubjetividad, al modo habermasiano. La posición antropológica, así entendida, no es una cuestión de todo o nada, sino que progresivamente se van viendo las implicaciones de su posición y se va desarrollando paralelamente a su exposición y al contraste con otras posiciones o situaciones diversas.

Aquí es donde descubrimos la función formativa de la Antropología de la Educación, ya que la percibimos entonces como un aprendizaje de la capacidad de pensar antropológicamente, lo que equivale a decir que valoramos los componentes argumentativos que son imprescindibles para adoptar y desarrollar una posición antropológica frente a cuestiones educativas.

Finalmente, desarrollar una posición antropológica respecto a cuestiones educativas no puede desvincularse de los cuatro apartados anteriores epistemológicos. Aprender a pensar con el objetivo de desarrollar una posición antropológica educativa significa adquirir una "visión antropológica" sobre Pedagogía. Y significa también usar herramientas antropológica, al abordar cuestiones pedagógicas. Ambas acepciones pueden aprenderse en contextos académicos, al depender, de modo significativo, de la calidad del entrenamiento antropológico recibido.

Aprender Antropología de la Educación es la única manera de pensar antropológicamente en educación; esto nos permitirá establecer algunas posiciones metodológicas más concretas sobre cómo realizar la enseñanza/aprendizaje en Antropología de la Educación:

$1^{\circ}$.Dicho aprendizaje se relaciona con lo que hemos calificado de "posiciones antropológicas particulares", es decir, con posiciones antropológicas explícitas sobre cuestiones pedagógicas. Las posiciones antropológicas particulares están, más o menos articuladas, en el contenido intelectual de la Antropología de la Educación. Lo cierto es que mediante el conocimiento y la adopción de posiciones antropológicas particulares, se puede desarrollar la posición antropológica personal. Por otra parte, la discusión de posiciones antropológicas particulares permite desarrollar un sentido fino del lenguaje antropológico y de los supuestos antropológicos implícitos en el lenguaje ordinario.

$2^{\circ}$.La posición antropológica se adquiere y desarrolla por la argumentación mediada intersubjetivamente. Por mediación intersubjetiva se entiende la discusión colectiva, la lectura de textos antropológicos y la exposición oral o escrita de argumentaciones antropológicas. No basta una lectura pasiva, sino que se exige una lectura entendida como diâlogo con los textos, conscientes de lo que acepta- 
HENRI BOUCHÉ PERIS, PACIANO FERMOSO ESTÉBANEZ, JORDI LARROSA BONDÍA, DAVID SACRISTÁN GÓMEZ (+) LA ANTROPOLOGÍA DE LA EDUCACIÓN COMO DISCIPLINA: PROYECTO DE DISEÑO

mos y de lo que rechazamos, porque la negativa a aceptar el contenido de un texto es también posicionarse. La intersubjetividad pide atender a los propios argumentos y a otros, que son críticos con nuestra posición, pues la posición antropológica no se conseguirá sin la "explicitación de las diferencias". Una posición antropológica es única, porque es distinta de otras y esto sólo se consigue mediante la explicación de las diferencias. (Dray: 1957).

$3^{\circ}$.La posición antropológica requiere un aprendizaje y percepción de las cuestiones educativas, aunque no se formulen expresamente desde perspectiva antropológica, pues en toda la Pedagogía late un corpus de ideas antropológicas. El análisis de las teorías antropológicas de la educación y el análisis de las diferentes pedagogías son buenos instrumentos para desarrollar este tipo de percepción antropológica.

$4^{\circ}$.La posición antropológica requiere ponerla a trabajar, o sea, procurar inscribir argumentaciones antropológicas en un campo determinado y, al mismo tiempo, abrir los distintos campos pedagógicos a la argumentación antropológica.

En resumen, la posición antropológica propuesta tiene como punto de partida la Antropología implicita, el trabajo con textos antropológicos educativos, la discusión y la argumentación, y el trabajo inmediato con la Antropología.

\section{Programas de Antropología de la Educación en las facultades españolas.}

El estatuto epistemológico de la Antropología de la Educación es el pórtico del nuevo edificio curricular, de reciente construcción en las Universidades españolas. Este pórtico nos permite hacer juicios de valor sobre nuestra realidad académica, desorientada en buena parte y arriesgada en otra, a la hora de llenar de contenidos el rótulo de una materia troncal en las nuevas titulaciones pedagógicas.

\section{Análisis de los contenidos de obras importantes en Antropología de la Educación.}

Previo al análisis empírico de los programas actuales de Antropología de la Educación, en algunas Universidades españolas, es orientativo, como referente comparativo, el verificado sobre los contenidos de algunas obras importantes de esta materia. Prescindiendo de los intentos de una "Antropología integral de la Educación", distinguimos tres grupos de obras:

$1^{\circ}$. Obras de Antropología Filosófica de la Educación, principalmente alemanas, en el periodo 1950-1989:

Bollnow, O.F. (1975): Die anthropologische Betranchtugs-weise in der Pädagogik,

Neur Detusche Schule Verlagsgesellschaft, Essen.

Dienelt, K. (1970): Antropología Pedagógica. Aguilar. Madrid.

Dienetl, K. (1984): Von der Matatheorie der Erziebung zur "sinn" orientierten Pädagogik. Diesterweg, Frankfurt a. Main.

Fermoso: P. (1984): Teoría de la Educación. Una interpretación antropológica. CEAC. Barcelona. 
Hamann, B. (1992): Antropología Pedagógica. Vicens Vives. Barcelona. Nohl, H. (1965): Antropología Pedagógica. F.C.E. México. Pleines, J. E.(1973): Pedagogik und Anthropologie. Henn, Ratingen.

\begin{tabular}{|l|c|c|c|c|c|c|c|c|c|c|c|}
\hline \multirow{2}{*}{ Autores } & \multicolumn{3}{|c|}{ Epistemología } & \multicolumn{6}{|c|}{ Corpus Básico Antropología Filosófica } \\
\cline { 2 - 17 } & A & B & C & D & E & F & G & H & I & J & $\mathrm{K}$ \\
\hline Bollnow & + & & + & & & + & & & & & + \\
\hline Dienelt (1970) & + & & & + & + & + & & & & & + \\
\hline Dienelt (1984) & + & & & + & & + & & & + & + & + \\
\hline Fermoso & + & & & + & + & + & & & & & + \\
\hline Hamann & + & & & + & & & & & & + & \\
\hline Nohl & & & & & & & + & + & & & \\
\hline Pleines & + & & & & & & & & & & \\
\hline & 6 & & 1 & 4 & 2 & 4 & 1 & 1 & 1 & 1 & 5 \\
\hline
\end{tabular}

A) Cuestiones Epistemológicas.

B) Antecedentes e Historia.

C) Métodos Heurísticos.

D) Imágen del hombre.

E) Comprensión del fenómeno educativo.

F) Antropología y Educación.

G) Formas de vida.

H) Estructura de la existencia humana.

I) Necesidad de la educación.

J) Conciencia y educación.

K) Modelos de antropología de la educación.

$2^{\circ}$. Obras de Antropología Cultural de la Educación principalmente las norteamericanas de "Educational Antbropology":

Brameld, Th. (1971): Bases culturales de la Educación. EUDEBA. Buenos Aires. García Castaño, J. y Pulido, R. (1949): Antropología de la Educación. Eudema, Madrid.

Kneller, G.F. (1974): Introducción a la Antropología Educacional. Paidós. Buenos Aires. 
Nicholson, C.K.(1969): Antropología y Educación. Paidós. Buenos Aires. Spindler, G.D. (1955): Education and Anthropology. Standford University.

\begin{tabular}{|l|c|c|c|c|c|c|c|c|c|c|}
\hline \multirow{2}{*}{ Autores } & \multicolumn{3}{|c|}{ Epistemología } & \multicolumn{6}{|c|}{ Corpus Básico Antropología Cultural } \\
\cline { 2 - 14 } & A & B & C & D & E & F & G & H & I & J \\
\hline Brameld & + & & + & & & + & + & + & & \\
\hline García C. & + & + & & & & + & & & & + \\
\hline Kneller & + & & & + & & & & & & \\
\hline Nicholson & + & + & & & & + & & & & \\
\hline Spindler & & + & & + & & & & + & + & + \\
\hline & 4 & 3 & 1 & 1 & 1 & 3 & 1 & 2 & 1 & 2 \\
\hline
\end{tabular}
A) Cultura: Conceptos, Clases...
B) Antecedentes e historia.
C) Etnografía.
D) Antropología y Educación.
E) Sociedades primitivas.
F) Adquisición y transmisión cultural.
G) Axiología y cultura.
H) Formación de maestros y antropología.
I) Funciones profesionales del antropólogo.
J) Modelos y concepciones.

$3^{\circ}$. Obras de Antropología Empírica, fundamentada en Ciencias Sociales:

La Antropología Empírica de la Educación tiene un excepcional representante en H. Roth, autor de: Pädagogische Anthropologie. Schroedel, Hannover, 1966. La obra tiene dos volúmenes:

Vol. $1^{\circ}$ : Bildsamkeit und Bestimmung (Plasticidad-formatividad y determinación). Estudia el ser inacabado del hombre, su indeterminación y la libertad de que disponer para terminarse de una u otra forma, es decir, para definirse y fijarse. Todo esto es posible gracias a la plasticidad humana.

Vol. 2: Entwicklung und Erziehung (Desarrollo y Educación). Estudia el ser evolutivo espacio-temporal del hombre y la educación. 


\section{2) Programas de Antropología de la Educación.}

A finales del curso académico 1993-94 hicimos una consulta epistolar a todos los Departamentos de Teoría e Historia de la Educación, de las Universidades españolas, para conocer, de facto, la situación y programación de la Antropología de la Educación en la nueva Licenciatura de Pedagogía. Muchas confirmaron su inexistencia, por no haber implantado aún dicha titulación — p.e., las Universidades de Murcia y de la Comunidad Autónoma de Galicia- o no estarse impartiendo su enseñanza por haberla ubicado en cursos superiores, aún no implantados. Respondieron afirmativamente y enviaron sus programas las Universidades de Barcelona, Autónoma de Barcelona, Rovira i Virgili de Tarragona, Complutense de Madrid y Málaga.

Principales Bloques Temáticos de estos Programas.

\begin{tabular}{|l|c|c|c|c|c|c|c|c|c|}
\hline \multirow{2}{*}{ Universidades } & \multicolumn{3}{|c|}{ Epistemología } & \multicolumn{3}{c|}{$\begin{array}{c}\text { Corpus Básico de } \\
\text { Antropología Filosófica }\end{array}$} & \multicolumn{2}{c|}{$\begin{array}{c}\text { Corpus Básico de } \\
\text { Antropología Cultural }\end{array}$} \\
\cline { 2 - 12 } & A & B & C & D & E & F & G & H & I \\
\hline Autó. Barna. & + & & + & & & & + & + & \\
\hline Barcelona $1^{\circ}$ & + & & + & & & + & & + & \\
\hline Complutense $1^{\circ}$ & + & & & + & + & & + & + & + \\
\hline Complutense $2^{\circ}$ & + & + & + & + & & & + & + & \\
\hline Complutense $3^{\circ}\left(^{*}\right)$ & & & & & & & & & \\
\hline Málaga & & & & + & & + & & + & \\
\hline Tarragona $1^{\circ}$ & + & + & + & + & & & & + & + \\
\hline Tarragona $2^{\circ}$ & + & + & & & & & + & + & + \\
\hline Tarragona $3^{\circ}$ & + & & & & & & + & & + \\
\hline Barcelona $2^{\circ}$ & + & + & & + & & & & + & \\
\hline Barcelona $3^{\circ}$ & & & & & & & & + & + \\
\hline & 8 & 4 & 4 & 5 & 1 & 2 & 5 & 9 & 5 \\
\hline
\end{tabular}
A) Conceptos fundamentales.
B) Métodos heurísticos.
C) Hombre y educación.
D) Supuestos antropológicos.
E) Desarrollo humano.

F) Educabilidad.

G) Modelos de antropología de la educación.

H) Cultura.

I) Multiculturalismo.

$\left(^{*}\right)$ El profesor Prieto desarrolla un programa atípico. 
Los profesores de esta materia son todos Titulares de Universidad: Jordi Larrosa, José L. Rodríguez y Violeta Núñez (Universidad de Barcelona), Joan Carles Mèlich (Autónoma de Barcelona), José $\mathrm{M}^{\mathrm{a}}$. Barrio, Gonzalo Jover y Rafael Prieto (Complutense de Madrid), Felipe Vega (Universidad de Málaga), Enric Fuentes, Isabel Miró y Luis F. Valero (Universidad Rovira i Virgili, de Tarragona). En total, once respuestas pioneras.

En estas respuestas existen varias coincidencias:

$1^{a}$. Denominación; "Antropología de la Educación", excepto una, que la califica de "Antropología Educativa".

$2^{a}$. Módulos: Tres son los más repetidos, tal vez por ser los sugeridos en el documento oficial del MEC, al imponer la troncalidad de esta materia, en la nueva Licenciatura en Pedagogía.

$3^{a}$. Profesorado: Titulares de Universidad, de los Departamentos de Teoría e Historia de la Educación, excepto el de la Diplomatura de "Educación Social" (Complutense de Madrid), que pertenece al de Sociología.

$4^{\mathrm{a}}$. Titulación: Es troncal en la Licenciatura de Pedagogía; pero se cursa también, como obligatoria u optativa de la propia Universidad en la Diplomatura de Educación Social (Complutense de Madrid y UNED) y en alguna especialidad de las siete de Magisterio (Rovira i Virgili, de Tarragona).

\section{3) Bibliografia coincidentemente citada.}

Diecinueve son las obras más repetidas en la Bibliografía, que acompaña a los programas enviados por los profesores de las Universidades españolas. En alguno la Bibliografía es muy abundante; pero hemos recogido únicamente aquellas obras que son citadas al menos en tres programas. Doce son las citadas únicamente en dos programas, sus autores: Almeida, Buber, Camilleri, Dearder, Dienelt, Freire, Jiménez Núñez, Jordán, Nicholson, Velasco, Woods y Zubiri.

* Obras citadas en tres programas:

Aselmeier, V. (1982): Antropología biológica y pedagógica, Alhambra, Madrid. Gehlen, A. (1993): Antropología Filosófica, Paidós, Barcelona.

Mead, M. (1985): Educación y cultura en Nueva Guinea, Paidós, Barcelona.

Scheuerl, H. (1985): Antropología Pedagógica, Herder, Barcelona.

* Obra citada cuatro veces:

Geertz, C. (1991): La interpretación de las culturas, Gedisa, Barcelona.

* Obras citadas cinco veces:

Hamann, B. (1992): Antropología Pedagógica, Vicens Vives, Barcelona. Harris, M. (1982): Introducción a la Antropología General, Alianza Editorial, Madrid. 
4) Principales Módulos de los programas.

Los Módulos están muy definidos y, en general, corresponden a las sugerencias hechas por el MEC y publicadas en el BOE. Los compaginamos con las indicaciones del MEC y recogemos los principales descriptores/materias.

* Módulo I: Cuestiones Epistemológicas.

- Indicaciones del MEC: "El discurso antropológico actual y el significado de una Antropología de la Educación. La investigación etnográfica escolar".

- Descriptores principales: Antropología de la Educación, Antropología Pedagógica, Clasificación de la Antropología, Conceptos básicos, Métodos de investigación en Antropología de la Educación.

* Módulo II: Supuestos Antropológicos de la Educación.

- Indicaciones del MEC: "El estudio del hombre en la Antropología actual. La educabilidad y la libertad. Educación y comunicación. Principales modelos pedagógico-antropológicos".

- Descriptores principales: Educabilidad, Imagen del hombre, Modelos pedagógico-antropológicos, Ser evolutivo del hombre, Ser inacabado y libre del hombre.

* Módulo III: Supuestos antropológicos de la cultura.

- Indicaciones del MEC: "Cultura y cambio cultural. La educación como enculturación. Teorías y ecosistemas de transmisión cultural. Ecología de las poblaciones humanas y desarrollo educativo".

- Descriptores principales: Aculturación y enculturación, Bilingüismo, Cultura y civilización, Cultura europea, Cultura internacional, Ecología, Etnografía, identidad y diversidad cultural, Multuculturalismo, Migraciones, Transmisión de Cultura.

\section{iII. Propuesta de Programa. Principales Módulos}

Para hacer una propuesta - no otra es nuestra intención y alcance- hemos conjugado: $1^{\circ}$ ) Indicaciones del MEC; $2^{\circ}$ ) Contenidos de las obras básicas; $3^{\circ}$ ) Programas facilitados por las Universidades españolas; $4^{\circ}$ ) Nuestro propio pensamiento, reflejado en la primera parte: "Estatuto epistemológico de la Antropología de la Educación".

\section{1) Cuestiones epistemológicas:}

* Antropología y Antropologías. La Antropología de la Educación en el contexto de los diversos estudios sobre el hombre.

* Concepto de Antropología de la Educación, por vía de negación: concep- 
ciones desacertadas o superadas. La Antropología de la Educación en el concierto de los saberes pedagógicos.

* Antecedentes de la Antropología de la Educación.

* Justificación y necesidad de la Antropología de la Educación.

* Principales modelos o concepciones de la Antropología de la Educación.

* Ambitos específicos de desarrollo de la Antropología de la Educación.

* Métodos de investigación en Antropología de la Educación.

\section{2) Antropología de la Educación desde la perspectiva filosófica:}

\section{Filosofía de la Educación}

La mayor parte de los descriptores de este Módulo se refieren al concepto de hombre y su proyección en la educación. Una fórmula genérica sería: "Fundamentos antropológicos de la educación". Desglosada esta proposición general, éstos son los contenidos del Módulo:

* Educabilidad y "homo educandus".

* Ser corporal y educación.

* Ser cognoscente y educación.

* Ser dialógico-referido; ser comunicable y comunicado, y educación.

* Ser inacabado, indeterminado, evolutivo, carente y abierto, y educación.

* Ser libre y educación.

3) Antropología de la Educación desde la perspectiva cultural y social: La Antropología cultural y social

* Adquisición y transmisión de cultura.

* Ecología de las poblaciones humanas y educación.

* El hombre, ser simbolizador y simbólico, producto y productor de cultura.

* Investigación etnográfica y educación.

* Multiculturalismo y educación en contextos democráticos. La identidad y diversidad culturales.

* Principales agentes culturales: ámbito familiar, ámbito institucional y escolar, ámbito sociopolítico.

* Procesos de aculturación y enculturación, y educación. 
BIBLIOGRAFÍA

AYeR, A. J. (1962): El problema del conocimiento, Eudeba, Buenos Aires.

Bertrán, M. (1982): "La Antropología Pedagógica", Perspectivas Pedagógicas, nro. 50 (1982), 221-222.

DrAY, W. (1957): Laws and explanation in bistory, Oxford Universty Press, London.

EsCÁmeZ, J. (1981): "Fundamentación antropológica de la educación", AA.VV.: Teoría de la Educación, Anaya, Madrid, p. 8 y ss.

Fermoso, P. (1994): "Nota histórico-bibliográfica sobre la Antropología Pedagógica en Norteamérica y Alemania", Anthropos, nro. 160 (1994), 78-85.

Foucault, M. (1973): El orden del discurso, Tusquets, Barcelona.

García-Castaño, J.- Pulddo, R.A. (1994): Antropología de la Educación, Eudema, Madrid.

HAGtroms (1965): The scientific community, Basic Books, New York.

Hamann, B. (1992): Antropología Pedagógica, Vicens Vives, Barcelona.

HuSSERL, E. (1942): Meditaciones cartesianas, F.C.E., México.

KanT, M. (1991): Sobre Pedagogía, Eumo, Vic.

Knorr-Cetina, K. D.- Mulay, M. (Eds.) (1983): Science observed. Perspectives on the social study of science, Sage, London.

KuHn, T. (1971): La estructura de las revoluciones científicas, F.C.E., México.

Mantovani, J. (1972): La educación y sus problemas, El Ateneo, Buenos Aires.

Nassif, R. (1979): Pedagogía General, Cincel, Madrid.

RADNISKY (1974): "Towards a systematic philosophy of scientific research", Pbilosophy of Social Sciences, 4 (1974).

RoRTY (1983): La filosofia y el espejo de la naturaleza, Cátedra, Madrid.

RoTh, H. (1966): Pädagogische Anthropologie, Schroedel, Hannover, 2 Vols.

SCHELER, M. (1971): El puesto del hombre en el cosmos, Losada, Buenos Aires.

WitLEY, R. (1984): The intelectual and social organization of sciences, Clarenton Press, Oxford.

ZDARZIL, H. (1975): "Pädagogische Anthropologie zwischen Empirie und Philosophie", Erziebung und Unterricht, nro. 125 (1975), 776-784. 